\title{
Prefronto-Cerebellar Transcranial Direct Current Stimulation Improves Sleep Quality in Euthymic Bipolar Patients: A Brief Report
}

\author{
Amedeo Minichino, Francesco Saverio Bersani, Francesco Spagnoli, \\ Alessandra Corrado, Francesco De Michele, Wanda Katharina Calò, \\ Martina Primavera, Baoran Yang, Laura Bernabei, Francesco Macrì, \\ Lucilla Vergnani, Massimo Biondi, and Roberto Delle Chiaie
}

Department of Neurology and Psychiatry, Sapienza University of Rome, Viale dell'Università 30, 00185 Rome, Italy

Correspondence should be addressed to Roberto Delle Chiaie; r.dellechiaie@centrokahlbaum.it

Received 25 August 2014; Revised 17 November 2014; Accepted 21 November 2014; Published 7 December 2014

Academic Editor: Gianfranco Spalletta

Copyright (c) 2014 Amedeo Minichino et al. This is an open access article distributed under the Creative Commons Attribution License, which permits unrestricted use, distribution, and reproduction in any medium, provided the original work is properly cited.

\begin{abstract}
Introduction. Sleep problems are common in bipolar disorder (BD) and may persist during the euthymic phase of the disease. The aim of the study was to improve sleep quality of euthymic BD patients through the administration of prefronto-cerebellar transcranial direct current stimulation (tDCS). Methods. 25 euthymic outpatients with a diagnosis of BD Type I or II have been enrolled in the study. tDCS montage was as follows: cathode on the right cerebellar cortex and anode over the left dorsolateral prefrontal cortex (DLPFC); the intensity of stimulation was set at $2 \mathrm{~mA}$ and delivered for $20 \mathrm{~min} /$ die for 3 consecutive weeks. The Pittsburgh Sleep Quality Index (PSQI) was used to assess sleep quality at baseline and after the tDCS treatment. Results. PSQI total score and all PSQI subdomains, with the exception of "sleep medication," significantly improved after treatment. Discussion. This is the first study where a positive effect of tDCS on the quality of sleep in euthymic BD patients has been reported. As both prefrontal cortex and cerebellum may play a role in regulating sleep processes, concomitant cathodal (inhibitory) stimulation of cerebellum and anodal (excitatory) stimulation of DLPFC may have the potential to modulate prefrontal-thalamic-cerebellar circuits leading to improvements of sleep quality.
\end{abstract}

\section{Introduction}

Sleep problems are common in bipolar disorder (BD). While it is well established that sleep disturbances are common features during manic and depressive episodes of the disorder, it is now starting to become clear that sleep dysfunctions are also common features in the euthymic phase of the disease $[1,2]$.

The course of BD has traditionally been viewed as episodic, with symptomatic and functional recovery between mood episodes. This view has recently been challenged by clinical and epidemiological studies that document how, despite symptomatic improvements or recovery following mood episodes, many BD individuals experience cognitive and social impairments even during the euthymic phase [3-5].
Recent studies hypothesized that these impairments could be related to the prefronto-thalamic-cerebellar circuit dysfunctions and, in particular, to the loss of the physiological inverse metabolic activity between dorsolateral prefrontal cortex (DLPFC) (hypoactivity) and subcortical areas such as thalamus and cerebellum (hyperactivity) [6-8]. Therefore, we performed a research study based on the administration of a prefrontal-cerebellar transcranial direct current stimulation (tDCS) protocol to euthymic BD patients in order to improve their neuropsychological functioning.

During the investigation, we noticed an unexpected and remarkable improvement in sleep quality in a large number of patients. We found this to be unexpected and of potential clinical relevance, as both the prefrontal cortex and cerebellum are known to play a role in modulating sleep processes 
$[9,10]$ and tDCS has the potential to induce hypomania and insomnia [11]. For these reasons, we performed the present subanalysis specifically centred on assessing the possible effectiveness of prefrontal-cerebellar tDCS on sleep quality.

\section{Methods}

2.1. Sample. The sample consisted of 25 outpatients ( 8 males; 17 females; mean age $41.9 \pm 12.62)$ with a diagnosis of $\mathrm{BD}$ Type I $(n=15)$ or II $(n=10)$ in the euthymic phase of the disease (disease duration $17.08 \pm 12.05$ years). Euthymic phase was defined as having a score of $<7$ on the Hamilton Depression Rating Scale (HDRS) and a score of $<7$ on the Young Mania Rating Scale (YMRS). Diagnoses were made through the Structured Clinical Interview for DSM-IV Axis I Disorders $(S C I D-I)$. Patients were consecutively recruited at the Policlinico Umberto I University Hospital of Rome. Patients were screened and excluded for having significant concomitant neurological or organic diseases, comorbid Axis I diagnoses, left handedness, pharmacological treatment with typical antipsychotics, or hospitalization in the last 12 months. All patients were on stable pharmacological treatment with lithium $(n=12)$ and/or anticonvulsants $(n=17)$ and/or atypical antipsychotics $(n=17)$ and/or benzodiazepines $(n=$ 10) and/or antidepressants $(n=5)$ for at least two months. tDCS was administered to patients in addition to the standard pharmacological maintenance therapies that they were already receiving; the said maintenance therapies remained unchanged.

2.2. tDCS. Brain modulating techniques are nowadays considered safe and effective options for the treatment of several neuropsychiatric conditions, including bipolar disorder; tDCS is a neuromodulating technique that delivers constant low, direct current to the brain area of interest via inhibitory (cathode) and excitatory (anode) electrodes [12-14]. tDCS was applied through two sponge electrodes (surface area $=$ $25 \mathrm{~cm}^{2}$ ) moistened with a saline solution. The electrode montage was as follows: cathodal tDCS on the right cerebellar cortex, $1 \mathrm{~cm}$ below and $4 \mathrm{~cm}$ lateral to the inion (approximately comparable to the projection of cerebellar lobule VII on the scalp), and anodal tDCS over the left DLPFC; electrode position was determined by the International 10/20 System for EEG Electrodes, such that Fp1 corresponded to the DLPFC. The onset and offset of the intervention involved current being gradually increased and decreased, respectively, over $10 \mathrm{~s}$. The intensity of stimulation was set at $2 \mathrm{~mA}$ and delivered for 20 min every working day for 3 consecutive weeks using a Magstim DC Stimulator Plus.

2.3. Sleep Assessment. We used the Pittsburgh Sleep Quality Index (PSQI), validated in the Italian language, to assess sleep at baseline and the day after the last tDCS session [15]. The PSQI is a self-administered questionnaire that assesses sleep quality and disturbances over a 30-day period. It consists of 19 self-rated questions grouped into seven clinically derived domains of sleep difficulties (sleep quality, sleep latency, sleep duration, habitual sleep efficiency, sleep disturbances, use of sleep medications, and daytime dysfunction). The seven
TABLE 1: PSQI total and subcomponents mean scores before and after treatment.

\begin{tabular}{lccc}
\hline & Before treatment & After treatment & $P$ \\
\hline Sleep quality & $1.52 \pm 1.04$ & $0.8 \pm 0.76$ & 0.007 \\
Sleep latency & $1.28 \pm 0.97$ & $0.76 \pm 0.59$ & 0.028 \\
Sleep duration & $1.36 \pm 0.90$ & $0.68 \pm 0.55$ & 0.002 \\
Sleep efficiency & $1 \pm 0.76$ & $0.64 \pm 0.48$ & 0.053 \\
$\begin{array}{l}\text { Sleep } \\
\text { disturbance }\end{array}$ & $1.64 \pm 0.95$ & $0.56 \pm 0.65$ & $<0.001$ \\
$\begin{array}{l}\text { Sleep } \\
\text { medication }\end{array}$ & $0.84 \pm 0.55$ & $0.84 \pm 0.55$ & 1 \\
$\begin{array}{l}\text { Daytime } \\
\text { dysfunction }\end{array}$ & $1.28 \pm 0.67$ & $0.56 \pm 0.50$ & $<0.001$ \\
$\begin{array}{l}\text { PSQI total } \\
\text { score }\end{array}$ & $8.92 \pm 3.30$ & $4.84 \pm 2.67$ & $<0.001$ \\
\hline
\end{tabular}

subcomponents correspond to specific dimensions related to sleep quality that are generally assessed in clinical practice. Each domain is rated equally on a Likert scale that ranges from 0 to 3 and is used to generate a global score of $0-21$; global PSQI scores $>5$ indicate poor overall sleep quality [15].

2.4. Statistical Analysis. Data were analyzed trough SPSS software version 20 . The normal distribution of data was assessed through the Shapiro-Wilk test. Student's $t$-test was used to compare PSQI scores at baseline and after tDCS treatment. All variables were assessed considering a significance level of $5 \%$.

\section{Results}

PSQI total scores and all PSQI subdomains, with the exception of "sleep medication," significantly improved after treatment (Table 1).

\section{Discussion}

BD patients (even in euthymic phase) exhibit a significantly worse sleep quality as compared with healthy controls $[1,2]$. To the best of our knowledge, this is the first paper in which a positive effect of tDCS on the quality of sleep in euthymic $\mathrm{BD}$ patients has been reported.

It has been suggested that sleep, cognitive, and affective dysregulation in $\mathrm{BD}$ might be related to overlapping neurobiological systems involving prefrontal cortex and its connections with deeper brain regions [9]. Independent studies, in fact, demonstrated that patients with $\mathrm{BD}$ in euthymic states and healthy individuals who underwent sleep deprivation presented similar cognitive and emotional deficits involving the prefrontal cortex [9], supporting the role of prefrontal top-down circuitry in concomitantly modulating cognitive, emotional, and sleep processes. In addition, it is known that the modulation of sleep may represent one of the nonmotor functions of the cerebellum $[10,16]$. Knowing this, it is reasonable to hypothesize that concomitant cathodal (inhibitory) stimulation of cerebellum and anodal (excitatory) stimulation of DLPFC may modulate these networks leading to improvements of sleep quality. 
Since pharmacological treatment remained unchanged during the stimulation protocol and all patients were on stable treatment for at least two months, this improvement cannot be attributed to the effects of medication. All patients tolerated tDCS without complications, supporting the idea that $\mathrm{tDCS}$ may represent an inexpensive, easy to administer, noninvasive, and painless treatment for sleep disturbances in psychiatric patients.

This study has several limitations including the small sample size, the absence of objective biological markers of sleep, and the absence of a placebo control group; in particular, the absence of placebo controls makes it very difficult to assess the impact of the intervention. Despite these limitations, this study represents a starting point in the study of the potential efficacy of prefrontal-cerebellar tDCS to improve sleep quality. Further studies on the topic are warranted.

\section{Conflict of Interests}

The authors declare that there is no conflict of interests regarding the publication of this paper.

\section{References}

[1] P. M. B. Rocha, F. S. Neves, and H. Corrêa, "Significant sleep disturbances in euthymic bipolar patients," Comprehensive Psychiatry, vol. 54, no. 7, pp. 1003-1008, 2013.

[2] F. S. Bersani, A. Iannitelli, F. Pacitti, and G. Bersani, "Sleep and biorythm disturbances in schizophrenia, mood and anxiety disorders: a review," Rivista di Psichiatria, vol. 47, no. 5, pp. 365-375, 2012.

[3] R. C. Kessler, H. S. Akiskal, M. Ames et al., "Prevalence and effects of mood disorders on work performance in a nationally representative sample of U.S. workers," The American Journal of Psychiatry, vol. 163, no. 9, pp. 1561-1568, 2006.

[4] C. D. Morris, D. J. Miklowitz, S. R. Wisniewski, A. A. Giese, M. R. Thomas, and M. H. Allen, "Care satisfaction, hope, and life functioning among adults with bipolar disorder: data from the first 1000 participants in the Systematic Treatment Enhancement Program," Comprehensive Psychiatry, vol. 46, no. 2, pp. 98104, 2005.

[5] G. Bersani, E. Polli, G. Valeriani et al., "Facial expression in patients with bipolar disorder and schizophrenia in response to emotional stimuli: a partially shared cognitive and social deficit of the two disorders," Neuropsychiatric Disease and Treatment, vol. 9, pp. 1137-1144, 2013.

[6] B. E. Benson, M. W. Willis, T. A. Ketter et al., "Interregional cerebral metabolic associativity during a continuous performance task (Part II) : differential alterations in bipolar and unipolar disorders," Psychiatry Research: Neuroimaging, vol. 164, no. 1, pp. 30-47, 2008.

[7] T. A. Kimbrell, T. A. Ketter, M. S. George et al., "Regional cerebral glucose utilization in patients with a range of severities of unipolar depression," Biological Psychiatry, vol. 51, no. 3, pp. 237-252, 2002.

[8] A. Minichino, F. S. Bersani, G. Trabucchi et al., "The role of cerebellum in unipolar and bipolar depression: a review of the main neurobiological findings," Rivista di Psichiatria, vol. 49, no. 3, pp. 124-131, 2014.

[9] B. S. McKenna and L. T. Eyler, "Overlapping prefrontal systems involved in cognitive and emotional processing in euthymic bipolar disorder and following sleep deprivation: a review of functional neuroimaging studies," Clinical Psychology Review, vol. 32, no. 7, pp. 650-663, 2012.

[10] L. M. DelRosso and R. Hoque, “The cerebellum and sleep," Neurologic Clinics, vol. 32, no. 4, pp. 893-900, 2014.

[11] V. Gálvez, A. Alonzo, D. Martin, P. B. Mitchell, P. Sachdev, and C. K. Loo, "Hypomania induction in a patient with bipolar II disorder by transcranial direct current stimulation (tDCS)," Journal of ECT, vol. 27, no. 3, pp. 256-258, 2011.

[12] C. Poreisz, K. Boros, A. Antal, and W. Paulus, "Safety aspects of transcranial direct current stimulation concerning healthy subjects and patients," Brain Research Bulletin, vol. 72, no. 4-6, pp. 208-214, 2007.

[13] L. F. Medeiros, I. C. de Souza, L. P. Vidor et al., "Neurobiological effects of transcranial direct current stimulation: a review," Frontiers in Psychiatry, vol. 3, article 110, 2012.

[14] F. S. Bersani and M. Biondi, "Historical recurrences in psychiatry: somatic therapies," Rivista di Psichiatria, vol. 47, no. 1, pp. 1-4, 2012.

[15] G. Curcio, D. Tempesta, S. Scarlata et al., "Validity of the Italian version of the Pittsburgh Sleep Quality Index (PSQI)," Neurological Sciences, vol. 34, no. 4, pp. 511-519, 2013.

[16] J. L. Pedroso, P. Braga-Neto, A. C. Felício et al., "Sleep disorders in cerebellar ataxias," Arquivos de Neuro-psiquiatria, vol. 69, no. 2A, pp. 253-257, 2011. 


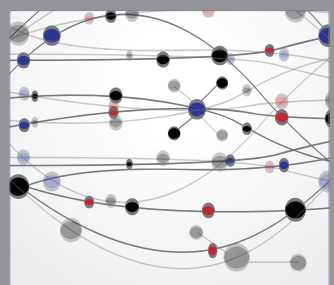

The Scientific World Journal
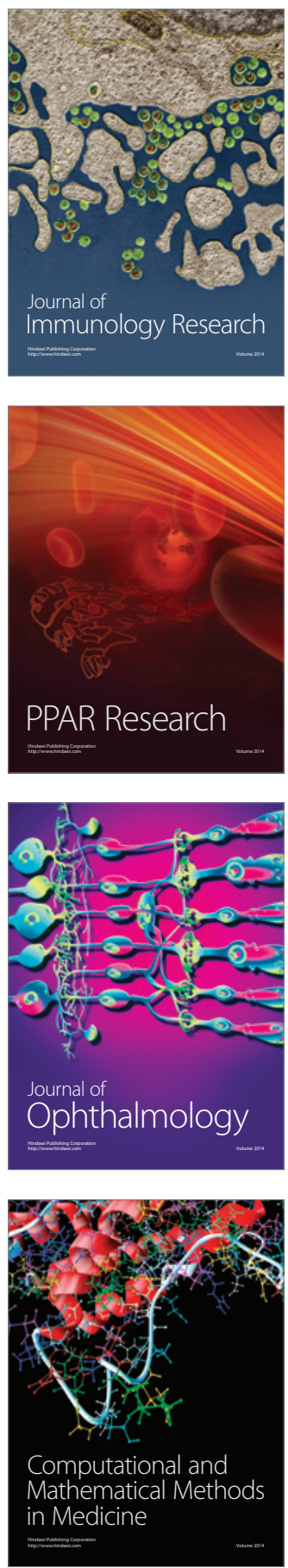

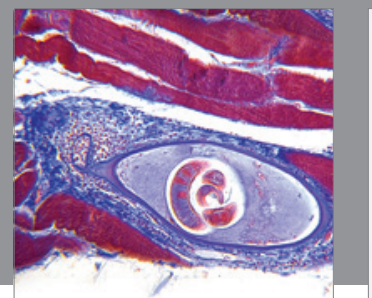

Gastroenterology

Research and Practice
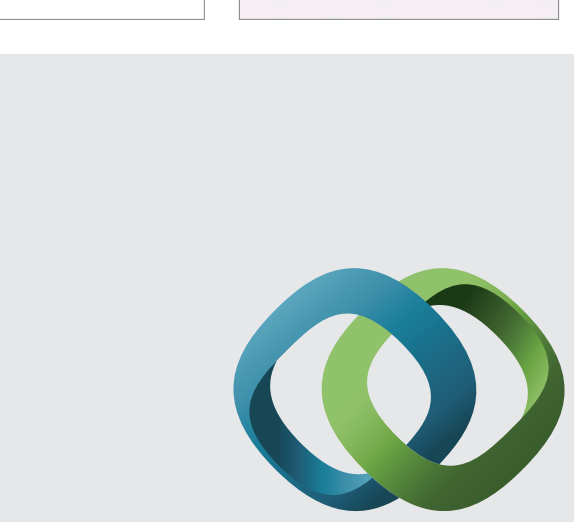

\section{Hindawi}

Submit your manuscripts at

http://www.hindawi.com

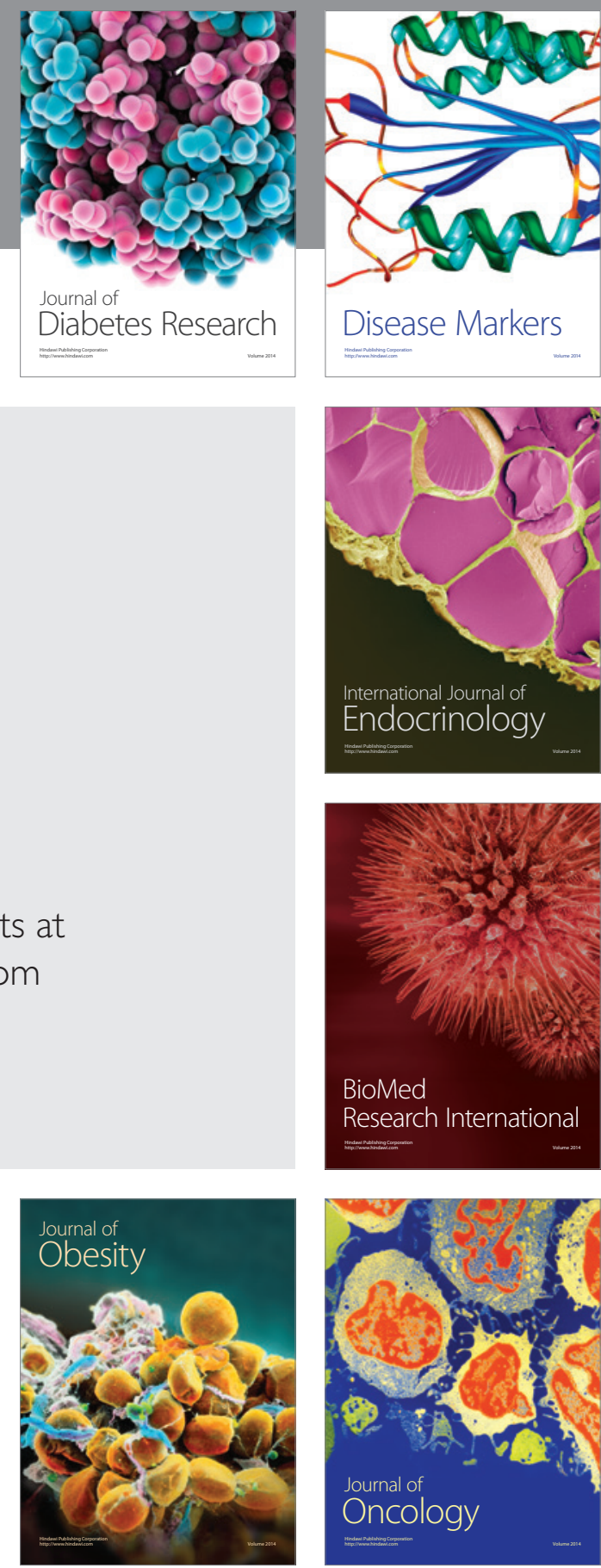

Disease Markers
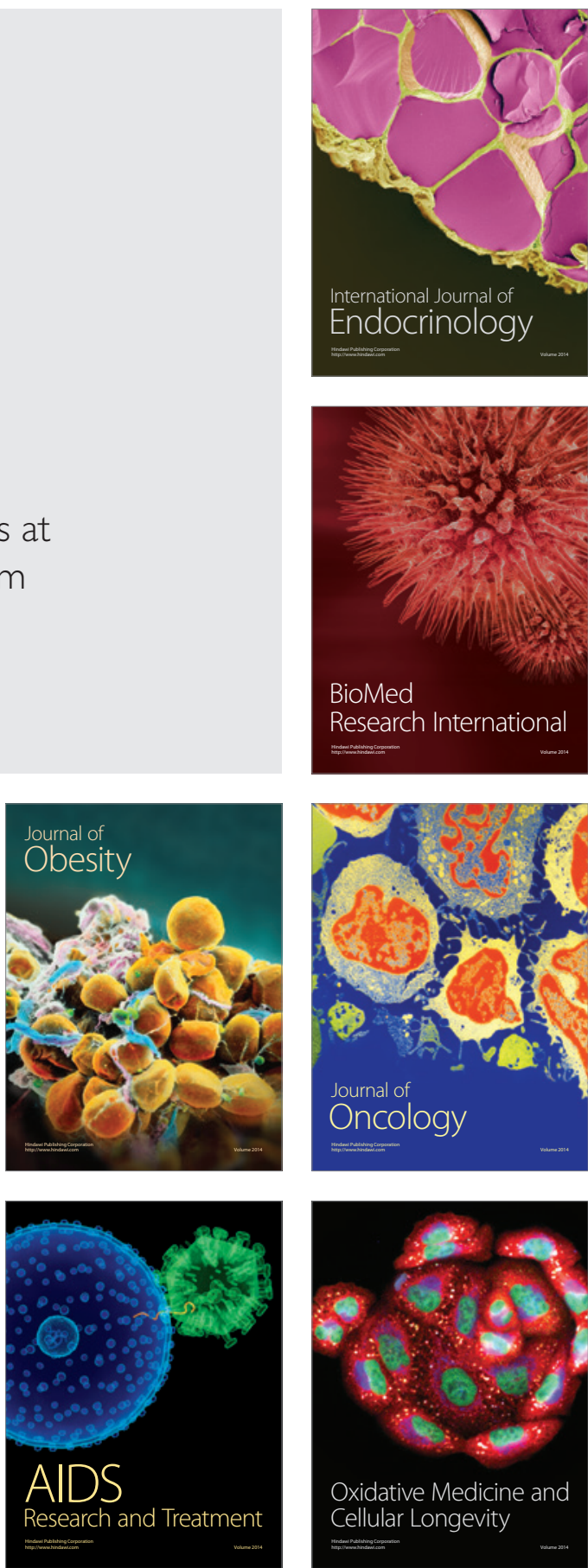